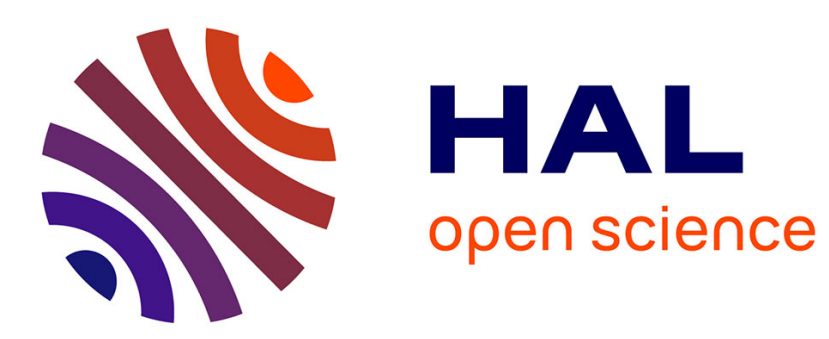

\title{
Optical Parabolic Pulse Generation and Applications
} Christophe Finot, John M. Dudley, Bertrand Kibler, D.J. Richardson, Guy

Millot

\section{To cite this version:}

Christophe Finot, John M. Dudley, Bertrand Kibler, D.J. Richardson, Guy Millot. Optical Parabolic Pulse Generation and Applications. IEEE Journal of Quantum Electronics, 2009, 45 (11), pp.14821489. 10.1109/JQE.2009.2027446 . hal-00447035

\section{HAL Id: hal-00447035 https://hal.science/hal-00447035}

Submitted on 27 Apr 2010

HAL is a multi-disciplinary open access archive for the deposit and dissemination of scientific research documents, whether they are published or not. The documents may come from teaching and research institutions in France or abroad, or from public or private research centers.
L'archive ouverte pluridisciplinaire HAL, est destinée au dépôt et à la diffusion de documents scientifiques de niveau recherche, publiés ou non, émanant des établissements d'enseignement et de recherche français ou étrangers, des laboratoires publics ou privés. 


\title{
Optical parabolic pulse generation and applications
}

\author{
C. Finot, J.M. Dudley, B. Kibler, D.J. Richardson and G. Millot
}

\begin{abstract}
Parabolic pulses in optical fibers have stimulated an increasing number of applications. We review here the physics underlying the generation of such pulses as well as the results obtained in a wide-range of experimental configurations.
\end{abstract}

Index Terms - Fiber amplifiers, Nonlinear dynamics, Optical pulse processing.

\section{INTRODUCTION}

$\mathrm{F}$ or more than three decades, optical fibers have been recognized as a versatile testbed with which to investigate a very wide range of nonlinear concepts. In 1980, Mollenauer et al. used optical single mode fibers to demonstrate the propagation of optical solitons [1] and the balance between linear anomalous dispersion and the Kerr nonlinearity of silica. More recently, parabolic pulse generation and evolution have motivated many studies [2]. Specifically, it has been shown that any pulse propagating in a normally dispersive fiber amplifier progressively reshapes into a pulse having a parabolic intensity profile combined with a perfectly linear chirp [3]. This new class of optical pulse is often referred to as a similariton pulse, and exhibits several fundamental features that are clearly attractive for applications ranging from high-power ultrashort pulse generation to optical nonlinear processing of telecommunication signals.

Although much of the background of this field is now wellknown, a number of important applications are becoming apparent that take particular advantage of the particular properties and features of this class of pulse. In this paper, we review a number of features of similariton pulse formation, as well as the wide range of experimental techniques that have been proposed to generate a parabolic intensity profile in both amplifying fibers as well as passive devices. Our intention is to provide a self-contained up-to-date overview of several specific aspects of this field that have not been reviewed in detail in previous studies. We also focus on some new applications that can benefit from the intrinsic properties of these pulses.

Manuscript received April 1, 2009

C. Finot, B. Kibler and G. Millot are with the Institut Carnot de Bourgogne, UMR 5209 CNRS-Université de Bourgogne, 9 Av. Alain Savary, BP 47870, 21078 Dijon, France. e-mail: christophe.finot@u-bourgogne.fr

J.M. Dudley is with the Institut FEMTO-ST, UMR 6174 CNRS-Université de Franche-Comté, Besançon, France.

D. Richardson is with the Optoelectronics Research Centre, Southampton University, Highfield Campus, Southampton, SO17 1BJ, United-Kingdom

\section{SElF-Similar Parabolic Pulses}

\section{A. Ultrashort pulse dynamics in a fiber amplifier}

During propagation in a fiber amplifier, gain, dispersion and nonlinearity interact together to strongly modify the properties of the initial ultrashort pulses. The resulting longitudinal evolution of the complex electric field envelope $\psi(z, T)$ can be quantitatively modeled in terms of the non-linear Schrödinger equation (NLSE) with a constant gain :

$$
i \frac{\partial \psi}{\partial z}=\frac{\beta_{2}}{2} \frac{\partial^{2} \psi}{\partial T^{2}}-\gamma|\psi|^{2} \psi+i \frac{g}{2} \psi
$$

with $\beta_{2}, \gamma$ and $g$ the second order dispersion, the Kerr nonlinear coefficient and the gain of the fiber, respectively.

Depending on the dispersion regime of the optical fiber, very different behaviors will then be observed. In the case of anomalous dispersion $\left(\beta_{2}<0\right)$, the pulses will undergo solitonlike compression [1] before splitting as a result of perturbed higher-order soliton dynamics [2]. On the other hand, in the case of normal dispersion $\left(\beta_{2}>0\right)$, the pulses will progressively broaden both in the temporal and spectral domains. More specifically, techniques based on the asymptotic self-similar analysis of this equation have outlined that, in the normal regime of dispersion, the evolution of any pulse can be accurately described in the limit $z \rightarrow \infty$ by the following asymptotic complex solution $\psi_{P}(z, T)[3]$ :

$$
\begin{cases}\psi_{P}(z, T)=A_{P}(z) \sqrt{1-\frac{T^{2}}{T_{P}^{2}(z)}} \exp \left(-i \frac{C_{P}}{2} T^{2}+i \varphi_{P}\right) & \text { if }|T| \leq T_{P} \\ \psi_{P}(z, T)=0 & \text { otherwise }\end{cases}
$$

This solution corresponds to a compactly-supported pulse with a parabolic intensity profile whose zero-crossing points are given by $T_{P}(z) . A_{P}(z), C_{P}$ and $\varphi_{P}$ are the amplitude of the pulse, the linear chirp slope and a constant phase offset, respectively. Their longitudinal evolution are explicitly given by the following set of equations first obtained by V.I. Kruglov [3-5]: 


$$
\left\{\begin{array}{l}
A_{P}(z)=\frac{1}{2} U_{i n i}^{1 / 3}\left(\frac{g}{\sqrt{\beta_{2} \gamma / 2}}\right)^{1 / 3} \exp \left(\frac{g}{3} z\right) \\
T_{P}(z)=3 U_{i n i}^{1 / 3} \frac{\sqrt[3]{\beta_{2} / 2}}{g^{2 / 3}} \exp \left(\frac{g}{3} z\right) \\
C_{P}=\frac{g}{3 \beta_{2}} \\
\varphi_{P}=\frac{3 \gamma A_{o}^{2}}{2 g} \exp \left(\frac{2 g z}{3}\right)
\end{array}\right.
$$

with $U_{i n i}$ being the initial pulse energy.

In the frequency domain, the corresponding asymptotic power spectrum is also parabolic with zero-crossing points of $\pm v_{p}(z)$ where $v_{P}(z)=C_{P} T_{P}(z)$.

In order to illustrate those analytical predictions, we have simulated the propagation of a Gaussian pulse having an initial duration of $40 \mathrm{ps}$ and an initial energy of $8 \mathrm{pJ}$. The pulse propagates in a $15-\mathrm{km}$ Raman amplifier based on a normally dispersive fiber with $\beta_{2}=16.610^{-3} \mathrm{ps}^{2} / \mathrm{m}$ and $\gamma=4 \mathrm{~W}^{-1} \cdot \mathrm{km}^{-1}$. The integrated gain of the amplifier is $27 \mathrm{~dB}$. Results of the numerical integration of the NLSE are plotted on Fig. 1. Figure 1(a) highlights the significant reshaping undergone by the initial Gaussian pulse which exhibits after propagation the expected parabolic shape. The temporal width and amplitude as well as the chirp of the pulse have been found to be in excellent agreement with the analytical results. When plotted on a semi-logarithmic scale, the intensity profile clearly reveals rapidly decreasing wings typical of the compact nature of the parabolic pulse. Some slowly decreasing wings can however be noticed below $10^{-15}$ and are residues of the preasymptotic evolution [6-8].
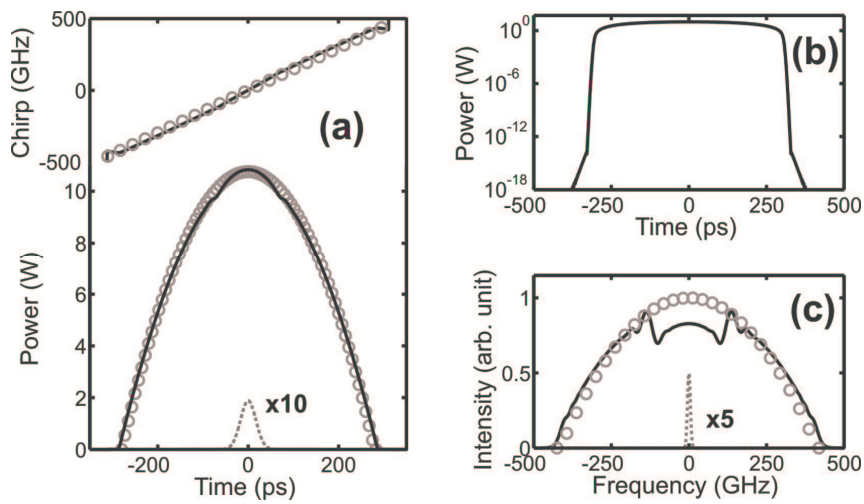

Fig. 1. (a) Temporal chirp and intensity profiles (b) Temporal intensity profile plotted on a logarithmic scale (c) Spectral intensity profile. The results of the numerical simulations (solid black lines) are compared with the analytical predictions (grey circles) and the initial pulse properties (dotted grey line)

Of course it is important to stress that the parabolic shape is efficiently generated only so long as the NLSE is a valid model to describe the pulse evolution in the fiber amplifier. Additional higher-order linear or nonlinear effects may indeed occur and ultimately limit the propagation length of the pulses. Among these deleterious effects, the Raman response of silica or the finite amplification bandwidth of the device have been shown to have relevant impact on the parabolic dynamics [912]. We also note that the constant gain assumption is not mandatory and parabolic pulses can also develop in the presence of longitudinal gain fluctuations $[4,13]$.

\section{B. A novel class of pulses : optical similaritons}

From the analytical expressions given by (2) and (3), we can identify several important features. First, once acquired, the parabolic shape is maintained as the pulse experiences exponential temporal and spectral broadening as well as an amplitude increase (see Fig. 2). In other words, the parabolic pulse is able to resist the deleterious effects of the wavebreaking $[2,8,14,15]$, a phenomenon which usually seriously degrades the evolution of any pulse evolving in a nonlinear normally dispersive medium [16-18]. As a consequence of this ability to propagate self-similarly, parabolic pulses are often referred to as optical similariton pulses [19].

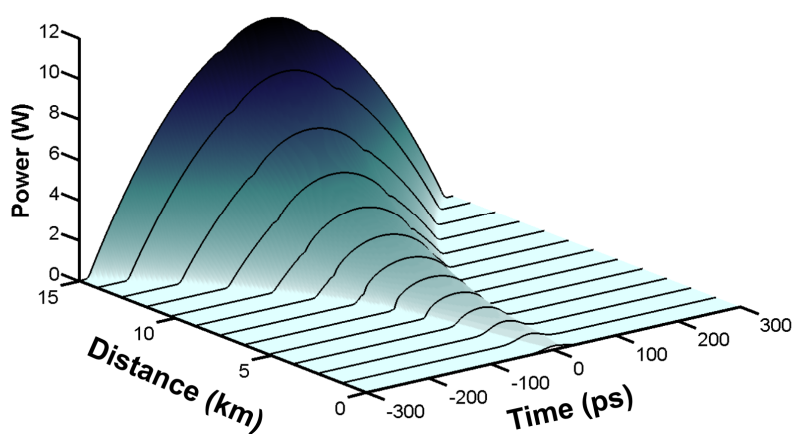

Fig. 2. Self-similar longitudinal evolution of the pulse in the normally dispersive Raman amplifier.

More remarkably, the output characteristics only depend on the energy of the initial pulse and are independent of the details of the initial intensity profile: the asymptotic state actually behaves as an attractor of the system [2, 20, 21]. In other words, initial pulses with significantly different temporal widths but with the same energy will converge after a sufficient propagation length in the amplifier towards the analytical state predicted by (2) and (3). Figure 3 illustrates this typical behavior : the longitudinal temporal evolutions of several initial pulses with identical energy but with temporal widths varying by a factor of more than ten are plotted on a logarithmic scale. As it can be seen, after a given propagation length which depends on the initial pulse properties, all the pulses undergo the exponential temporal broadening predicted by Eq. (3). The problem of choosing the optimum initial pulse property in order to shorten the propagation length required to enter into the asymptotic parabolic regime has been the subject of several studies [7, 18, 21-24] that have stressed the interactions between dispersion and nonlinearity in the initial stages of propagation. 

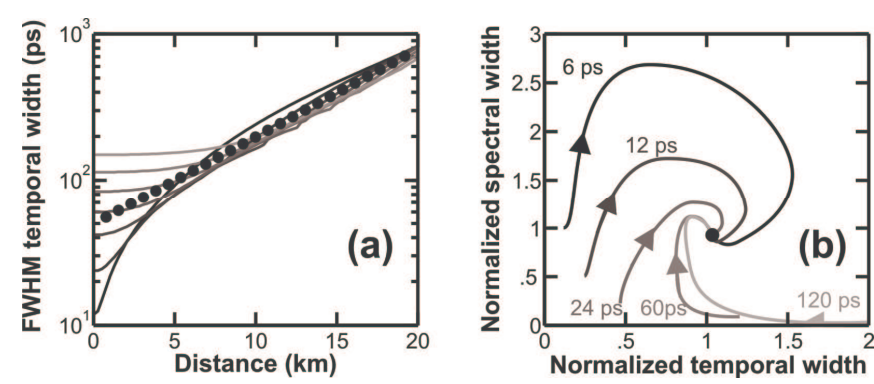

Fig. 3. Evolution of the pulse parameters towards the asymptotic parabolic solution: (a) Longitudinal evolution of the temporal full width at half maximum of various pulses with different initial duration compared with the solution given by (3) (plotted with solid black circles) (b) Different trajectories in the phase space for various initial pulse durations. For each point of the trajectory, temporal and spectral widths are normalized with respects to the temporal and spectral widths predicted by (2) and (3).

The dynamics of a pair of optical similaritons with the same or different central wavelengths has also been theoretically and experimentally investigated [25] : similaritons are robust against collisions [26], whereas the interaction of similaritons leads to the generation of high-repetition rate dark soliton trains [27]. Evolution of dark structures embedded on compact parabolic background has also been the subject of intense recent theoretical studies [28-31].

\section{Extension of optical similaritons to other pulse shapes and other nonlinear media}

The interest in parabolic self-similar pulses has not been restricted to the configuration of a single lumped amplifier, and the potential of similariton pulse shaping in fiber cavities has been demonstrated numerically as early as 2001 [25]. Additional experiments and theoretical studies have lead to a brand new class of high power femtosecond mode-locked oscillators [32, 33]. Compared to soliton lasers or dispersionmanaged cavities, similariton pulses have been shown to yield significant improvements in terms of pulse energy [34, 35]. Although there are many competing technologies based on alternative fiber designs, similariton lasers (like similariton amplifiers) are particularly attractive for the simplicity of their experimental realization.

Although parabolic pulses have been the first kind of optical pulses to be clearly identified as having a self-similar dynamics, other pulse shapes can also maintain their typical shape unchanged during propagation in an optical nonlinear fiber. More specifically, it has been shown that under certain conditions, optical fibers with longitudinally varying parameters can sustain the propagation of exact self-similar pulses having chirped hyperbolic secant or hyperbolic tangent profiles for example [36-40]. Other recent theoretical studies have also successfully investigated the possibility to extend the concept of self-similar bright and dark optical structures to birefringent fibers [41, 42], to optical non linear waveguides with a graded index profile $[28,30,31,43-46]$ as well as to two- or three- dimensional optical beam propagation $[47,48]$.

\section{EXPERIMENTAL GENERATION}

\section{A. Generation in active media}

The first clear experimental demonstration of the theoretical predictions of Eq. (2) and (3) have been carried out in the framework of ytterbium-doped fiber amplifiers [3]. A precise intensity and phase measurement based on the frequency resolved optical gating (FROG) definitively confirmed the parabolic intensity profile of the emerging picosecond pulses, as well as the high linearity of the chirp. Since then, several other experiments have benefited of the parabolic features in ytterbium doped fibers with increasing output power levels and various initial femtosecond seeds from passively modelocked fiber lasers with repetition rates of a few tens of $\mathrm{MHz}$ [3] to VECSELs with $\mathrm{GHz}$ repetition rates [49]. Since the pulse reshaping process is not restricted to a specific rare-earth dopant, generation in erbium-doped amplifiers at telecommunication wavelengths has also been successfully tested $[8,50,51]$. Indeed, it should be noted that as soon as 1996, experiments in erbium doped fiber done by Tamura et al. provided indirect evidence of the chirp linearity of pulses amplified in a normally dispersive fiber [52]. At this time, however, the self-similar nature of the parabolic pulses and their underlying fundamental properties were not clearly pointed out.
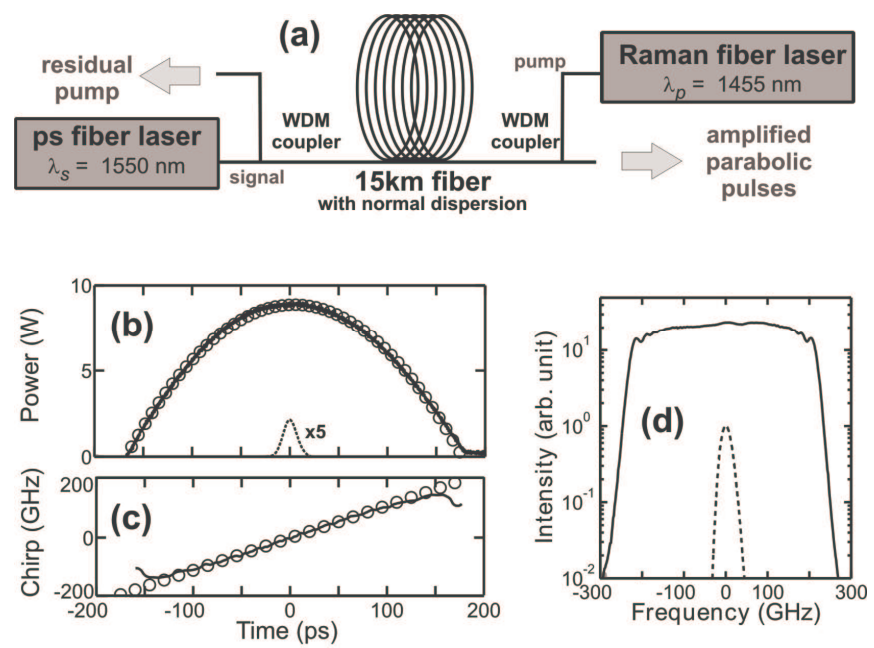

Fig. 4. Generation of parabolic pulses through Raman amplification at telecommunication wavelength $\quad$ (a) Experimental set-up $\quad$ (b) Intensity profiles of the initial pulse (dotted line) and output pulse (solid black line) compared with a parabolic fit (circles) $\quad$ (c) Chirp of the output pulse compared with a linear fit (circles) $\quad$ (d) Spectrum of the initial and output pulses (dashed and solid lines respectively). Results adapted from [53].

Other gain mechanisms are also fully suitable for asymptotic parabolic generation and configurations based on the use of the Raman amplification effect have been successfully investigated [53, 54]. As an illustration, Fig. 4 presents the setup and results of an experiment corresponding to numerical simulations presented in section II where similaritons are generated through Raman amplification at telecommunication wavelengths. In this all-fiber set-up using only standard devices, initial 14-ps pulses are adiabatically amplified in a 15$\mathrm{km}$ normally dispersive fiber and the output pulse intensity 
profile is recorded in real-time by a high-speed photodetector and oscilloscope. The emerging 200-ps pulses clearly exhibit the expected parabolic intensity profile combined with a highly linear chirp. The output optical spectrum also highlights the clear reshaping of the pulse, with a low ripple in the central part and a rapid decrease of the wings. This then confirms that parabolic pulses can be efficiently generated over a wide range of initial pulse durations (from 100 fs to $10 \mathrm{ps)} \mathrm{and} \mathrm{fiber}$ lengths (from a few meters to several kilometers).

\section{B. Generation in passive fibers relying on a nonlinear dynamics}

Active similariton amplifiers have found great success, but it can be also of interest to study alternative generation methods, especially in the context of optical telecommunications or for wavelengths for which adequate rare earth dopants or pumps are not available. Several specific techniques have been recently experimentally demonstrated. Based on the observation that a longitudinal decrease of the normal dispersion is formally equivalent to optical gain, it has for example been proposed to use continuously tapered dispersion decreasing with a dispersion profile exponentially decreasing down to 0 [55]. However, third order dispersion and optical attenuation have been found to ultimately limit the device performance $[56,57]$. Using tapering down to nonzero (normal) dispersion values with possibly additional Raman pumping can overcome those limitations [58]. A potential drawback of the method based on dispersion decreasing fibers is that such tapered fibers are not to date commercially available. Fortunately, the use of comb-like structures made of several segments with fixed dispersion has been found to efficiently mimic a continuous dispersion decrease [59].
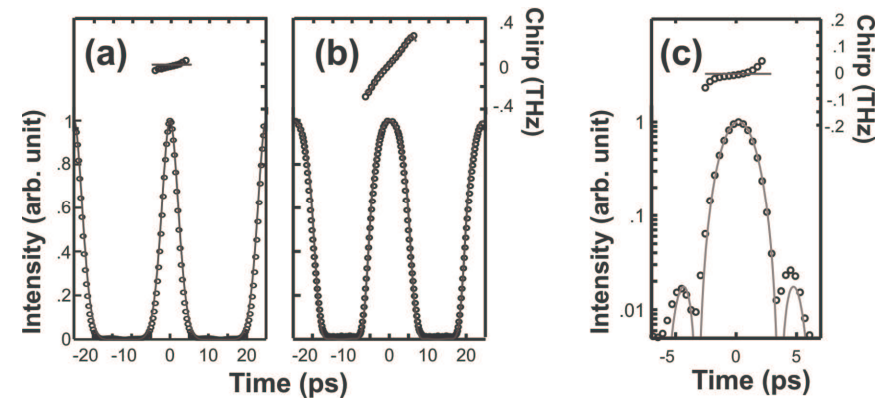

Fig. 5. Nonlinear passive reshaping observed in a two segment configuration. Initial Gaussian pulse train at a repetition rate of $40 \mathrm{GHz}$ (a) is reshaped into parabolic pulse train (b) after propagation in a normal dispersion shifted fiber followed by a highly nonlinear fiber. Experimental results obtained by FROG characterization (empty circles) are compared with the numerical integration of the NLSE (solid line). $\quad$ (c) Details of parabolic pulse after linear compression (results recorded for a repetition rate of $20 \mathrm{GHz}$ ). Experimental results (circles) are compared with a first order Bessel function of the first kind. Results adapted from reference [60].

Recent observations have led to another approach taking advantage of the progressive nonlinear reshaping of a pulse propagating in a nonlinear fiber with fixed dispersion [61]. Indeed, it has been shown that for a given initial pulse shape with precise input parameters, the temporal profile obtained after a carefully chosen propagation distance is in close agreement with a parabolic intensity profile combined with a nearly linear chirp [24, 62]. There are however some fundamental differences when compared to the previously described parabolic pulses in the sense that the pulse generated by this latter technique are not able to maintain their characteristic shape during further propagation. In order to stabilize their parabolic features, one has to use a second stage with higher nonlinearity and less dispersion [24, 63]. Figure 5 illustrates such an approach that has successfully been used to reshape an initial Gaussian pulse train at a repetition rate of 40 $\mathrm{GHz}$ into a train of parabolic pulses [60].

\section{Generation based on linear pulse shaping techniques}

All the previously described generation methods are based on the intrinsic nonlinear reshaping of an optical pulse propagating in an active or passive normally dispersive fiber. It is of course also possible to benefit from the progress achieved in the field of linear pulse shaping. This removes the need for a high initial peak power, which is especially beneficial in the context of telecommunication applications at high repetition rates where the required average power could be prohibitive. We note, however, that whereas the nonlinear passive reshaping does not induce losses, the linear techniques are intrinsically lossy.

Three approaches have been to date reported. The first approach relies on the use of a superstructured fiber Bragg grating to tailor the initial spectrum of picosecond pulses [6466]. Contrary to the nonlinear methods, it is here possible to record chirp-free parabolic intensity profiles. A second flexible approach takes advantage of the line by line shaping in an arrayed waveguide grating $[67,68]$ to produce bright or dark parabolic pulse trains. A third shaping scheme that has been recently proposed is based on the use of long period fiber grating filter [69].

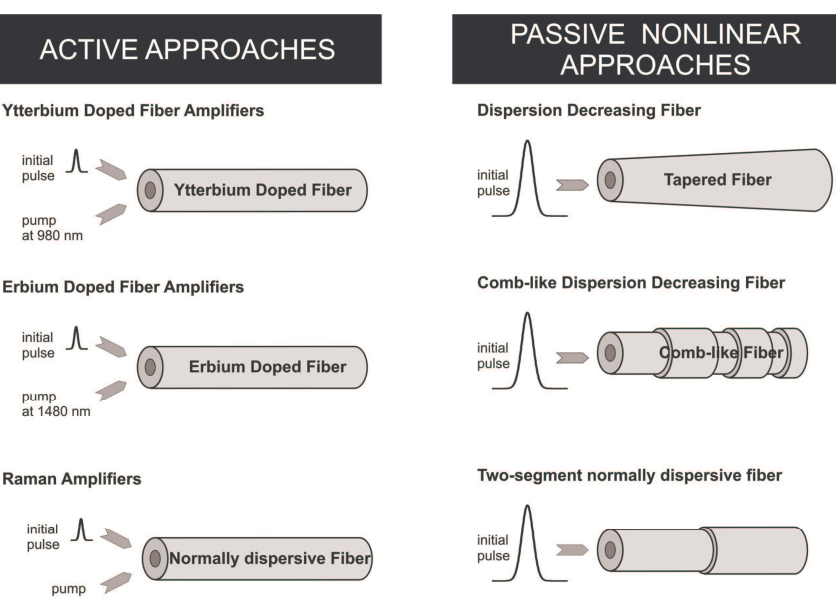

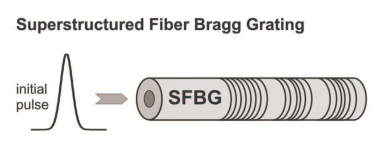

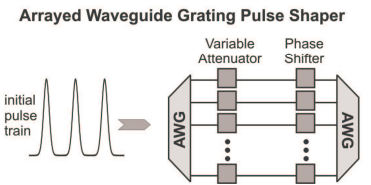

Fig. 6. Various active and passive approaches for parabolic pulse generation 


\section{APPLICATIONS}

Optical similaritons are not only of theoretical interest but they have very wide practical potential. The remarkable features of parabolic pulses discussed above have indeed stimulated numerous applications both in ultrashort high-power pulse generation and in optical pulse processing.

\section{A. High Power pulse amplification and ultrashort pulse generation}

The field of ultrashort high-power pulse generation has strongly benefited from the highly linear chirp of the amplified pulses [3, 49]. Indeed, the direct nonlinear amplification of pulses avoids the necessity of a prechirping stage as required in the linear chirped pulse amplification approach [70] where the ultimate goal is to avoid any nonlinearity by stretching the initial pulse and therefore reducing the initial peak power. The approach based on parabolic amplification is intrinsically different, as it takes advantage of the nonlinear optical spectrum expansion of the pulse. Indeed, a strong frequency chirp develops during amplification. Thanks to the high level of linearity of this chirp, it is possible to efficiently compensate for the positive chirp slope by using a pair of diffraction gratings so that high quality recompressed pulses can be obtained with final temporal durations much shorter than the initial seed pulse. Indeed, the theoretical pulse shape obtained after compression of a perfectly parabolic pulse is a first order Bessel function of the first kind that exhibits noticeably low sidelobes [23] (see Fig. 5(c) for experimentally compressed parabolic pulses compared to a first order Bessel function fit ; the sidelobe level is more than $18 \mathrm{~dB}$ below the peak power). Therefore, several experimental works have reported high performance results [8, 71-73], with temporal widths below 100 fs and peak-powers exceeding 4 MW [74] or pulse energy at the microjoule level [71]. Improvements in the technique have led to the use of cubicon pulses where the effect of residual third order dispersion can be overcome by an initial pulse shaping $[75,76]$. Parabolic amplification can also sustain $\mathrm{GHz}$ repetition rates $[49,51]$ and can be integrated in an all-fiber set-up with the judicious use of hollow-core photonic bandgap fibers employed as compression stages [5]. Additional nonlinear higher-order soliton compression has enabled the generation of pulses with temporal durations as short as $20 \mathrm{fs}$ (i.e. four optical cycles at $1550 \mathrm{~nm}$ ) [77]. If the primary goal is not to reach high peak powers, a length of anomalously dispersive fiber can be used [60, 62, 64, 69].

\section{B. Highly coherent continuums for optical telecommunications}

Highly coherent pulse generation has also taken advantage of the parabolic pulses ability to resist the effects of optical wavebreaking. Continuum with low spectral ripple and high energy spectral density in the central part can indeed be achieved [64] and the temporal coherence of the continuum obtained in the normal dispersion regime has been found superior to the case of continuum generated in the anomalous regime in the presence of solitonic effects or modulational instability. Such features can be of great interest for applications such as pulse shaping [78] or multiwavelength picosecond sources. Examples of low-noise multichannel sources running at 10 $\mathrm{GHz}$ and covering the whole C-band of the optical telecommunications have therefore been demonstrated based on km-long erbium doped fiber amplifier [51] or on the passive propagation of a parabolic pulse in a highly non-linear fiber $[64,69]$.

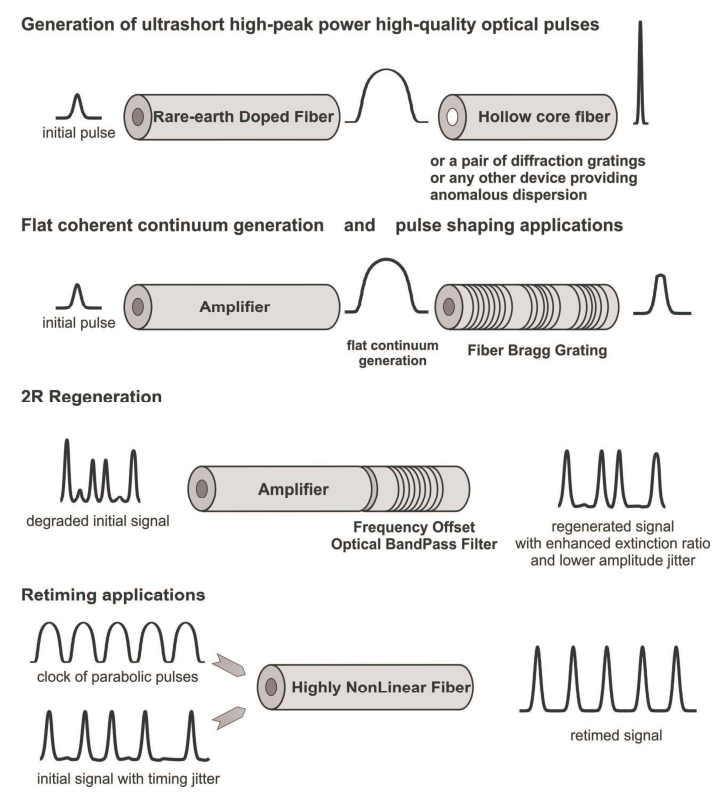

Fig. 7. Various applications of parabolic pulses

\section{Ultrafast all-optical signal processing}

In the high-bit rate telecommunication context [19], using parabolic pulses has also enabled noticeable improvements of existing optical pulse processing techniques based on the quasi-instantaneous Kerr response of highly nonlinear optical fibers. Various applications targeting the optical regeneration of an optical signal impaired by amplitude or timing jitter have been proposed.

In an extension of the technique initially proposed by P.V. Mamyshev [79], distributed Raman amplification in a normally dispersive fiber followed by offset spectral filtering has been shown to improve the ability of a $2 \mathrm{R}$ regenerator to eliminate spurious noise pulses as well as to simultaneously reduce any fluctuations in the signal ' 1 ' bit level [80].

Given their typical parabolic intensity profiles, the crossphase modulation (XPM) induced by a parabolic pulse onto another pulse is also strictly parabolic. This feature is of great interest for several optical high-speed applications where electro-optic phase modulators are not available, or are unable to provide a sufficient phase shift. Therefore, taking advantage of the perfectly linear chirp that can be generated through XPM induced by a parabolic pulse, it is possible to increase the level of tolerable temporal jitter that an optical retiming system can accommodate [65]. More recently, parabolic phase profiles induced by XPM have also been shown to be beneficial for the restoration of the intensity profile of pulses degraded by linear dispersive effects. Indeed, the time-domain optical Fourier transformation approach is based on imparting 
a highly linear chirp to the distorted data bits [81] and the use of parabolic pulses represents an attractive solution to generate the required chirp for picosecond pulse signals [66, 68]. We also anticipate that in the near future other applications, such as those based on time-lens effects, will benefit from such an approach.

\section{CONCLUSION}

In conclusion, parabolic self-similar pulses represent a new class of ultrashort optical pulses that have opened new avenues of fundamental and applied photonics research. A wide set of active or passive techniques has progressively become available to generate these pulses for applications such as ultrashort high-power pulse generation, highly coherent continuum sources and optical signal processing in high-bit rate telecommunications, to name just a few.

\section{ACKNOWLEDGMENTS}

We would like to acknowledge the significant scientific contributions of S. Pitois, J. Fatome, K. Hammani, S. Wabnitz (Institut CARNOT de Bourgogne), P. Dupriez, F. Parmigiani, L. Provost, J.H.V. Price, M. Ibsen, P. Petropoulos (Optoelectronics Research Centre).

\section{REFERENCES}

[1] L. F. Mollenauer, R. H. Stolen, and J. P. Gordon, "Experimental observation of picosecond pulse narrowing and solitons in optical fibers," Phys. Rev. Lett., vol. 45, pp. 1095-1098, 1980.

[2] J. M. Dudley, C. Finot, G. Millot, and D. J. Richardson, "Self-similarity in ultrafast nonlinear optics," Nat. Phys., vol. 3, pp. 597-603, 2007.

[3] M. E. Fermann, V. I. Kruglov, B. C. Thomsen, J. M. Dudley, and J. D. Harvey, "Self-similar propagation and amplification of parabolic pulses in optical fibers," Phys. Rev. Lett., vol. 84, pp. 6010-6013, 2000.

[4] V. I. Kruglov, A. C. Peacock, J. M. Dudley, and J. D. Harvey, "Selfsimilar propagation of high-power parabolic pulses in optical fiber amplifiers," Opt. Lett., vol. 25, pp. 1753-1755, 2000.

[5] V. I. Kruglov, A. C. Peacock, J. D. Harvey, and J. M. Dudley, "Selfsimilar propagation of parabolic pulses in normal-dispersion fiber amplifiers," J. Opt. Soc. Amer. B, vol. 19, pp. 461-469, 2002.

[6] S. Boscolo, S. K. Turitsyn, V. Y. Novokshenov, and J. H. B. Nijhof, "Self-similar parabolic optical solitary waves," Theor. Math. Phys., vol. 133, pp. 1647-1656, 2002.

[7] V. I. Kruglov and J. D. Harvey, "Asymptotically exact parabolic solutions of the generalized nonlinear Schrödinger equation with varying parameters," J. Opt. Soc. Amer. B, vol. 23, pp. 2541-2550, 2006.

[8] C. Billet, J. M. Dudley, N. Joly, and J. C. Knight, "Intermediate asymptotic evolution and photonic bandgap fiber compression of optical similaritons around $1550 \mathrm{~nm}, "$ Opt. Express, vol. 13, pp. 3236-3241, 2005.

[9] A. C. Peacock, R. J. Kruhlak, J. D. Harvey, and J. M. Dudley, "Solitary pulse propagation in high gain optical fiber amplifiers with normal group velocity dispersion," Opt. Commun., vol. 206, pp. 171-177, 2002.

[10]D. B. S. Soh, J. Nilsson, and A. B. Grudinin, "Efficient femtosecond pulse generation using parabolic amplifier combined with a pulse compressor. I. Stimulated Raman-scattering effects," J. Opt. Soc. Amer. $B$, vol. 23 , pp. 1-9, 2006.

[11]D. B. S. Soh, J. Nilsson, and A. B. Grudinin, "Efficient femtosecond pulse generation using a parabolic amplifier combined with a pulse compressor. II. Finite gain-bandwidth effect," J. Opt. Soc. Am. B, vol. 23, pp. 10-19, 2006
[12] G. Chang, A. Galvanauskas, H. G. Winful, and T. B. Norris, "Dependence of parabolic pulse amplification on stimulated Raman scattering and gain bandwith," Opt. Lett., vol. 29, pp. 2647-2549, 2004.

[13]C. Finot, "Influence of the pumping configuration on the generation of optical similaritons in optical fibers," Opt. Commun., vol. 249, pp. 553$561,2005$.

[14]D. Anderson, M. Desaix, M. Karlson, M. Lisak, and M. L. QuirogaTeixeiro, "Wave-breaking-free pulses in nonlinear optical fibers," J. Opt. Soc. Am. B, vol. 10, pp. 1185-1190, 1993.

[15] C. Finot, G. Millot, S. Pitois, C. Billet, and J. M. Dudley, "Numerical and experimental study of parabolic pulses generated via Raman amplification in standard optical fibers," IEEE J. Sel. Top. Quantum Electron., vol. 10, pp. 1211-1218, 2004.

[16] W. J. Tomlinson, R. H. Stolen, and A. M. Johnson, "Optical wavebreaking of pulses in nonlinear optical fibers," Opt. Lett., vol. 10, pp. 457-459, 1985.

[17]D. Anderson, M. Desaix, M. Lisak, and M. L. Quiroga-Teixeiro, "Wavebreaking in nonlinear optical fibers," J. Opt. Soc. Am. B, vol. 9, pp. 13581361,1992

[18] C. Finot, B. Kibler, L. Provost, and S. Wabnitz, "Beneficial impact of wave-breaking or coherent continuum formation in normally dispersive nonlinear fibers," J. Opt. Soc. Am. B, vol. 25, pp. 1938-1948, 2008.

[19] M. E. Fermann, B. C. Thomsen, V. I. Kruglov, J. M. Dudley, and J. D. Harvey, "Parabolic pulse communication," US Patent, 2001.

[20]C. Finot, G. Millot, and J. M. Dudley, "Asymptotic characteristics of parabolic similariton pulses in optical fiber amplifiers," Opt. Lett., vol. 29, pp. 2533-2535, 2004.

[21]S. Wabnitz, "Analytical Dynamics of parabolic pulses in nonlinear optical fiber amplifiers," IEEE Photon. Technol. Lett., vol. 19, pp. 507$509,2007$.

[22] Y. Ozeki, Y. Takushima, K. Taira, and K. Kikuchi, "Clean similariton generation from an initial pulse optimized by the backward propagation method," in Conference on Lasers and Electro-Optics (CLEO US), 2004, pp. CTuBB51113-1114.

[23] C. Finot, F. Parmigiani, P. Petropoulos, and D. J. Richardson, "Parabolic pulse evolution in normally dispersive fiber amplifiers preceding the similariton formation regime," Opt. Express, vol. 14, pp. 3161-3170, 2006.

[24] C. Finot, L. Provost, P. Petropoulos, and D. J. Richardson, "Parabolic pulse generation through passive nonlinear pulse reshaping in a normally dispersive two segment fiber device," Opt. Express, vol. 15, pp. 852-864, 2007.

[25] A. C. Peacock, V. I. Kruglov, B. C. Thomsen, J. D. Harvey, M. E. Fermann, G. Sucha, D. Harter, and J. M. Dudley, "Generation and interaction of parabolic pulses in high gain fiber amplifiers and oscillators.," in Optical Fiber Communication Conference (OFC), Anhei, California, 2001, pp. 1-3.

[26] C. Finot and G. Millot, "Collisions of optical similaritons," Opt. Express, vol. 13, pp. 7653-7665, 2005.

[27]C. Finot and G. Millot, "Interactions of optical similaritons," Opt. Express, vol. 13, pp. 5825-5830, 2005.

[28] L. Wu, J.-F. Zhang, L. Li, C. Finot, and K. Porsezian, "Similaritons interaction in nonlinear graded-index waveguide amplifiers," Phys. Rev. A, vol. 78, p. 053807, 2008.

[29] L. Wu, J.-F. Zhang, C. Finot, and L. Li, "Propagation of dark similariton on the compact parabolic background in dispersion-managed optical fibers," Opt. Express, vol. 17, pp. 8278-8286, 2009.

[30]L. Li, X. Zhao, and Z. Xu, "Dark solitons on an intense parabolic background in nonlinear waveguides," Phys. Rev. A, vol. 78, p. 063833 , 2008.

[31]X. Zhao, L. Li, and Z. Xu, "Dark-soliton stripes on a paraboloidal background in a bulk nonlinear medium," Phys. Rev. A, vol. 79, p. 043827, 2009

[32]F. Ö. Ilday, J. R. Buckley, W. G. Clark, and F. W. Wise, "Self-similar evolution of parabolic pulses in a laser," Phys. Rev. Lett., vol. 92, p. 213902, 2004.

[33] A. Ruehl, O. Prochnow, D. Wandt, D. Kracht, B. Burgoyne, N. Godbout, and S. Lacroix, "Dynamics of parabolic pulses in an ultrafast fiber laser," Opt. Lett., vol. 31, pp. 2734-2736, 2006.

[34]F. Wise, "Similariton lasers generate high-energy femtosecond pulses," in Optics \& Photonics News, 2004, p. 45. 
[35]A. Ruehl, D. Wandt, U. Morgner, and D. Kracht, "Normal dispersive ultrafast fiber oscillators," IEEE J. Sel. Top. Quantum Electron., vol. 15, pp. 170-181, 2009.

[36] S. A. Ponomarenko and G. P. Agrawal, "Interactions of chirped and chirp-free similaritons in optical fiber amplifiers," Opt. Express, vol. 15, pp. 2963-2973, 2007

[37] V. I. Kruglov, A. C. Peacock, and J. D. Harvey, "Exact self-similar solutions of the generalized nonlinear Schrödinger equation with distributed coefficients," Phys. Rev. Lett., vol. 90, p. 113902, 2004.

[38] D. Méchin, S. H. Im, V. I. Kruglov, and J. D. Harvey, "Experimental demonstration of similariton pulse compression in a comblike dispersiondecreasing fiber amplifier," Opt. Lett., vol. 31, pp. 2106-2108, 2006.

[39] S. Chen, L. Yi, G. Dong-Sheng, and P. Lu, "Self-similar evolutions of parabolic, Hermite-Gaussian, and hybrid optical pulses : Universality and diversity," Phys. Rev. E, vol. 72, p. 016622, 2005.

[40] V. I. Kruglov, D. Méchin, and J. D. Harvey, "Self-similar solutions of the generalized Schrödinger equation with distributed coefficients," Opt. Express, vol. 12, pp. 6198-6207, 2004.

[41] V. I. Kruglov, D. Méchin, and J. D. Harvey, "Parabolic and quasiparabolic two-component coupled propagating regimes in optical amplifiers," Phys. Rev. A, vol. 77, p. 033846, 2008.

[42] G. Chang, H. G. Winful, A. Galvanauskas, and T. B. Norris, "Incoherent self-similarities of the coupled amplified nonlinear Schrödinger equations," Phys. Rev. E, vol. 73, p. 016616, 2006.

[43] L. Wu, L. Li, and J.-F. Zhang, "Controllable generation and propagation of asymptotic parabolic optical waves in graded-index waveguide amplifiers," Phys. Rev. A, vol. 78, 2008.

[44] L. Wu, J.-F. Zhang, L. Li, Q. Tian, and K. Porsezian, "Similaritons in nonlinear optical systems," Opt. Express, vol. 16, pp. 6352-6360, 2008.

[45] S. A. Ponomarenko and G. P. Agrawal, "Do solitonlike self-similar waves exist in nonlinear optical media ?," Phys. Rev. Lett., vol. 97, p. 013901, 2006.

[46] S. A. Ponomarenko and G. P. Agrawal, "Optical similaritons in nonlinear waveguide amplifiers," Opt. Lett., 2007.

[47] G. Chang, H. G. Winful, A. Galvanauskas, and T. B. Norris, "Self-similar parabolic beam generation and propagation," Phys. Rev. E, vol. 72, p. 016609,2005

[48] S. Chen and J. M. Dudley, "Spatiotemporal nonlinear optical selfsimilarity in three dimensions," Phys. Rev. Lett., vol. 102, p. 233903, 2009.

[49]P. Dupriez, C. Finot, A. Malinowski, J. K. Sahu, J. Nilsson, D. J. Richardson, K. G. Wilcox, H. D. Foreman, and A. C. Tropper, "Highpower, high repetition rate picosecond and femtosecond sources based on Yb-doped fiber amplification of VECSELS," Opt. Express, vol. 14, pp. 9611-9616, 2006.

[50] J. W. Nicholson, A. Yablon, P. S. Westbrook, K. S. Feder, and M. F. Yan, "High power, single mode, all-fiber source of femtosecond pulses at $1550 \mathrm{~nm}$ and its use in supercontinuum generation," Opt. Express, vol. 12, pp. 3025-3034, 2004.

[51] Y. Ozeki, Y. Takushima, K. Aiso, and K. Kikuchi, "High repetition-rate similariton generation in normal dispersion erbium-doped fiber amplifiers and its application to multi-wavelength light sources," IEICE Trans. Electron., vol. 88, pp. 904-911, 2005.

[52] K. Tamura and M. Nakazawa, "Pulse compression by nonlinear pulse evolution with reduced optical wave breaking in erbium-doped fiber amplifiers," Opt. Lett., vol. 21, pp. 68-70, 1996.

[53] K. Hammani, C. Finot, S. Pitois, J. Fatome, and G. Millot, "Real time measurement of long parabolic optical similaritons," Electron. Lett., vol. 44, pp. 1239-1240, 2008

[54]C. Finot, G. Millot, C. Billet, and J. M. Dudley, "Experimental generation of parabolic pulses via Raman amplification in optical fiber," Opt. Express, vol. 11, pp. 1547-1552, 2003.

[55]T. Hirooka and M. Nakazawa, "Parabolic pulse generation by use of a dispersion-decreasing fiber with normal group-velocity dispersion," Opt. Lett., vol. 29, pp. 498-500, 2004.

[56] A. Latkin, S. K. Turitsyn, and A. Sysoliatin, "On the theory of parabolic pulse generation in tapered fibre," Opt. Lett., vol. 32, pp. 331-333, 2007.

[57]C. Zhang, G. Zhao, A. Luo, and Z. Zhang, "Third-order dispersion role on parabolic pulse propagation in dispersion-decreasing fiber with normal group-velocity dispersion," Appl. Phys. B, 2008.
[58] C. Finot, B. Barviau, G. Millot, A. Guryanov, A. Sysoliatin, and S. Wabnitz, "Parabolic pulse generation with active or passive dispersion decreasing optical fibers," Opt. Express, vol. 15, pp. 15824-15835, 2007.

[59]B. Kibler, C. Billet, P. A. Lacourt, R. Ferrière, L. Larger, and J. M. Dudley, "Parabolic pulse generation in comb-like profiled dispersion decreasing fibre," Electron. Lett., vol. 42, pp. 965-966 2006.

[60] C. Finot, J. Fatome, S. Pitois, and G. Millot, "All-Fibered High-Quality Low Duty-Cycle 20-GHz and 40-GHz Picosecond Pulse Sources," IEEE Photon. Technol. Lett., vol. 19, pp. 1711-1713, 2007.

[61] A. Zeytunyan, G. Yesayan, L. Mouradian, P. Kockaert, P. Emplit, F. Louradour, and A. Barthélémy, "Nonlinear-dispersive similariton of passive fiber," J. Europ. Opt. Soc. Rap. Public., vol. 4, p. 09009, 2009.

[62] S. Pitois, C. Finot, J. Fatome, and G. Millot, "Generation of 20-Ghz picosecond pulse trains in the normal and anomalous dispersion regimes of optical fibers," Opt. Commun., vol. 260, pp. 301-306, 2006.

[63] S. Boscolo, A. I. Latkin, and S. K. Turitsyn, "Passive nonlinear pulse shaping in normally dispersive fiber systems," IEEE J. Quantum Electron., vol. 44, pp. 1196-1203, 2008.

[64]F. Parmigiani, C. Finot, K. Mukasa, M. Ibsen, M. A. F. Roelens, P. Petropoulos, and D. J. Richardson, "Ultra-flat SPM-broadened spectra in a highly nonlinear fiber using parabolic pulses formed in a fiber Bragg grating," Opt. Express, vol. 14, pp. 7617-7622, 2006.

[65]F. Parmigiani, P. Petropoulos, M. Ibsen, and D. J. Richardson, "Pulse retiming based on XPM using parabolic pulses formed in a fiber Bragg grating," IEEE Photon. Technol. Lett., vol. 18, pp. 829-831, 2006.

[66] T. T. Ng, F. Parmigiani, M. Ibsen, Z. Zhang, P. Petropoulos, and D. J. Richardson, "Compensation of linear distortions by using XPM with parabolic pulses as a time lens," IEEE Photon. Technol. Lett., vol. 20, pp. 1097-1099, 2008.

[67] T. Hirooka, M. Nakazawa, and K. Okamoto, "Bright and dark $40 \mathrm{GHz}$ parabolic pulse generation using a picosecond optical pulse train and an arrayed waveguide grating," Opt. Lett., vol. 33, 2008.

[68] T. Hirooka and M. Nakazawa, "All-optical 40-GHz Time-Domain Fourier Transformation using XPM with a dark parabolic pulse," IEEE Photon. Technol. Lett., vol. 20, pp. 1869-1871, 2008.

[69]D. Krcmarik, R. Slavik, Y. Park, and J. Azana, "Nonlinear pulse compression of picosecond parabolic-like pulses synthesized with a long period fiber grating filter," Opt. Express, vol. 17, pp. 7074-7087, 2009.

[70]D. Strickland and G. Mourou, "Compression of amplified chirped optical pulses," Opt. Commun., vol. 56, pp. 219-221, 1985.

[71] T. Schreiber, C. K. Nielsen, B. Ortac, J. P. Limpert, and A. Tünnermann, "Microjoule-level all-polarization-maintaining femtosecond fiber source," Opt. Lett., vol. 31, pp. 574-576, 2006.

[72] A. Malinowski, A. Piper, J. H. V. Price, K. Furusawa, Y. Jeong, J. Nilsson, and D. J. Richardson, "Ultrashort-pulse Yb3+ fiber based laser and amplifier system producing > $25 \mathrm{~W}$ average power," Opt. Lett., vol. 29, pp. 2073-2075, 2004.

[73] J. P. Limpert, T. Schreiber, T. Clausnitzer, K. Zöllner, H. J. Fuchs, E. B. Kley, H. Zellmer, and A. Tünnermann, "High-power femtosecond Ybdoped fiber amplifier," Opt. Express, vol. 10, pp. 628-638, 2002.

[74]D. N. Papadopoulos, Y. Zaouter, M. Hanna, F. Druon, E. Mottay, E. Cormier, and P. Georges, "Generation of 63 fs 4.1 MW peak power pulses from a parabolic fiber amplifier operated beyond the gain bandwith limit," Opt. Lett., vol. 32, pp. 2520-2522, 2007.

[75] L. Shah, Z. Liu, I. Hartl, G. Imeshev, G. C. Cho, and M. E. Fermann, "High energy femtosecond $\mathrm{Yb}$ cubicon fiber amplifier," Opt. Express, vol. 13, pp. 4717-4722, 2005.

[76] S. Zhou, L. Kuznetsova, A. Chong, and F. W. Wise, "Compensation of nonlinear phase shifts with third-order dispersion in short-pulse fiber amplifiers," Opt. Express, vol. 13, pp. 4869-4877, 2005.

[77] B. Kibler, C. Billet, P. A. Lacourt, R. Ferrière, and J. M. Dudley, "Allfiber source of 20 -fs pulses at $1550 \mathrm{~nm}$ using two-stage linear-nonlinear compression of parabolic similaritons," IEEE Photon. Technol. Lett., vol. 18, pp. 1831-1833, 2006.

[78]C. Finot and G. Millot, "Synthesis of optical pulses by use of similaritons," Opt. Express, vol. 12, pp. 5104-5109, 2004.

[79]P. V. Mamyshev, "All-optical data regeneration based on self-phase modulation effect," in European Conference on Optical Communication, ECOC'98, Institute of Electrical and Electronics Engineering, Madrid, Spain, 1998, pp. 475-476. 
[80]C. Finot, S. Pitois, and G. Millot, "Regenerative 40-Gb/s wavelength converter based on similariton generation," Opt. Lett., vol. 30, pp. 1776$1778,2005$.

[81]T. Hirooka and M. Nakazawa, "Optical adaptative equalization of highspeed signals using time-domain optical Fourier transformation," $J$. Lightw. Technol., vol. 24, pp. 2530-2540, 2006. 